\title{
Are you sure that it is a drug allergy?
}

\author{
Eun Hee Chung, MD, PhD \\ Department of Pediatrics, Chungnam National University School of Medicine, Daejeon, Korea
}

Adverse drug reactions (ADRs) include 2 types, namely types A and B. Type A ADRs include overdose toxicity, commonly observed side effects, indirect effects, and drug-drug interactions based on a drug's inherent pharmacological action. Type B ADRs include intolerance, drug idiosyncrasy, drug hypersensitivity reactions (DHRs) caused by immunological mechanisms and pseudoallergic reactions caused by nonimmune mechanisms. ${ }^{1)}$ A DHR is a drug-mediated immunological mechanism in sensitized patients and occurs in a narrow sense in combination with drug allergies (DAs). ${ }^{2)}$

The clinical manifestation of DA is variable; sometimes children already have various diseases when suspected allergic symptoms occur. In children, respiratory and gastrointestinal symptoms, along with skin symptoms, are the most common allergic symptoms, which are like those of the underlying disease for which the drug is administered. Therefore, clinicians may miss some DAs. Moreover, clinicians diagnose DAs according to drug administration history alone without performing a diag. nostic evaluation.

Drug provocation tests (DPTs) and skin tests were previously performed for suspected DAs in 43 children who were assessed by physicians to have DHRs. Among them, however, DHRs were confirmed only in 3 patients. ${ }^{3)}$ The DPTs were positive in about $25 \%-30 \%$ of children with suspected DHR. ${ }^{4,5)}$ These results differed from those suspected clinically and those confirmed by diagnostic evaluation. The diagnosis of DA based on medical history alone is vague and unreliable, leading to overdiagnosis. Limiting the prescriptions of drugs causing suspected DAs without a definite diagnosis restricts the number of alternative drugs, especially in children who have fewer approved options than adults. It is important to accurately diagnose a DA because difficulty selecting appropriate drugs can result in less effective treatment and the need for subsequent treatment, which in turn can increase medical costs.

This review of drug allergies in children ${ }^{6}$ highlights a wide range of topics, including DA epidemiology, the causative agent, and diagnostic methods. When diagnosing DA, history taking and examination are important to determining suspected diseases and drugs. Furthermore, objective tests such as skin tests, DPTs, and serum tests are performed. To accurately diagnose
DA, it is necessary to confirm that it is a symptom of an immunological mechanism of a drug response. However, unlike tests for determining the causes of other allergies, tests to measure serum-specific immunoglobulin $\mathrm{E}$ (IgE) for drugs in DAs are not commercially available, except for specific IgE for a few drugs. ${ }^{6}$ Some in vitro tests (basophil activation test and lymphocyte transformation test) are difficult to perform in clinical practice. The most commonly used methods for DA diagnosis are skin tests and DPT. However, the skin prick test and intradermal test have limitations that a negative result can occur in cases of a non-IgE-mediated ADR. Intradermal tests cannot be easily performed in children due to pain and difficulty. Therefore, DPTs are useful in the diagnosis of DA in children. ${ }^{4-6)}$

The major causative drugs of ADR in children are antibiotics, especially beta-lactams; nonsteroidal anti-inflammatory drugs; and vaccines. ${ }^{6,7)}$ DAs can be suspected when a symptom develops after drug administration, and the suspected drug can be selected and subjected to further evaluation. It is important to distinguish between immediate and nonimmediate reactions by checking the time to symptom onset after drug administration. While selecting a diagnostic test method, history taking is important. A detailed history in patients with a suspected DA was well documented in this review, which may be of great help in clinical practice.

When a DA is suspected, it is important to pinpoint the cause, the identification of which can help prevent the development of allergic symptoms. It is also important to be aware of DA and avoid prescribing medications that cause allergies. Although the diagnosis of DA depends on medical history and there are few standardized tests, identifying the causative agent of DA is very important in preventing its occurrence. In this regard, the review of Park and Suh ${ }^{6}$ explains the current knowledge of DA in children, which seems to be of great help in its diagnosis and prevention in pediatric patients.

See the article "Drug allergy in children: what should we know?” via https://doi.org/10.3345/kjp.2019.00675.

Corresponding author: Eun Hee Chung, MD, PhD. Department of Pediatrics, Chungnam National University Hospital, 282 Munwha-ro, Jung-gu, Daejeon 35015, Korea 凶E-mail: ehchung@cnu.ac.kr, http://orcid.org/0000-0001-9380-0151

Received: 30 November, 2019, Revised: 30 January, 2020, Accepted: 26 February, 2020

This is an open-access article distributed under the terms of the Creative Commons Attribution Non-Commercial License (http://creativecommons.org/licenses/bync/4.0/) which permits unrestricted non-commercial use, distribution, and reproduction in any medium, provided the original work is properly cited.

Copyright (c) 2020 by The Korean Pediatric Society 


\section{References}

1. Wheatley LM, Plaut M, Schwaninger JM, Banerji A, Castells M, Finkelman FD, et al. Report from the National Institute of Allergy and Infectious Diseases workshop on drug allergy. J Allergy Clin Immunol 2015;136:262-71.e2.

2. Joint Task Force on Practice Parameters; American Academy of Allergy, Asthma and Immunology; American College of Allergy, Asthma and Immunology; Joint Council of Allergy, Asthma and Immunology. Drug allergy: an updated practice parameter. Ann Allergy Asthma Immunol 2010;105:259-73.

3. Seitz CS, Bröcker EB, Trautmann A. Diagnosis of drug hypersensitivity in children and adolescents: discrepancy between physician-based assessment and results of testing. Pediatr Allergy Immunol 2011;22:405-10.

4. Choi J, Lee JY, Kim KH, Choi J, Ahn K, Kim J. Evaluation of drug provocation tests in Korean children: a single center experience. Asian Pac J Allergy Immunol 2016;34:130-6.

5. Noh SR, Yoon J, Cho HJ, Song S, Park GM, Yu J, et al. Outcomes of drug provocation test in Korean children with suspected drug hypersensitivity reaction. Allergy Asthma Respir Dis 2017;6:26-33.

6. Park JS, Suh DI. Drug allergy in children: what should we know? Clin Exp Pediatr 2020 Feb 6 [Epub]. https://doi.org/10.3345/kjp.2019.00675.

7. Chung EH. Vaccine allergies. Clin Exp Vaccine Res 2014;3:50-7. 